\title{
Newsfronts
}

\section{Transgenic Pigs Make Xenotransplantation More Kosher}

The recent creation of pigs whose cells lack certain sugar molecules may bring the dream of xenotransplantation a step closer to reality. At first glance, $\alpha 1,3$-galactosyltransferase $(\alpha 1,3 \mathrm{GT})$ appears to be a fairly innocuous enzyme; in most mammalian species, $\alpha 1,3 \mathrm{GT}$ participates in the production of carbohydrate chains that adorn cell surfaces in a wide variety of tissues.

However, evolutionary events from millions of years ago resulted in the elimination of $\alpha 1,3 \mathrm{GT}$ expression in Old World monkeys, apes, and humans, and the $\alpha 1,3 \mathrm{Gal}$ surface epitopes produced by this protein represent a primary obstacle to successful xenotransplantation. These carbohydrates trigger a significant human immune response; thus, the transplantation of tissues into humans from pigs or other donor species is generally doomed to failure as a result of hyperacute rejection (HAR), wherein the host immune system attacks and rapidly destroys the offending foreign tissue.

Researchers at PPL Therapeutics (Blacksburg, VA) and the University of Pittsburgh Medical Center (PA) took an important step toward reducing the threat of HAR by creating a line of pigs that lack functional $\alpha 1,3 \mathrm{GT}$ (Science, online 19 December 2003). The group isolated fetal cells from a previously developed transgenic line with one functional copy of the $\alpha 1,3 \mathrm{GT}$ gene, then used Clostridium difficile toxin A, which specifically kills cells producing $\alpha 1,3 \mathrm{Gal}$ epitopes, to isolate lines completely lacking $\alpha 1,3 \mathrm{GT}$ activity.

Apparently a spontaneous mutation abrogating enzyme function eliminated the second gene copy, and the researchers used the selected cell lines to generate four knockout piglets. $\alpha 1,3 \mathrm{Gal}$ was undetectable in various tissues from these piglets, and pancreatic islet cells transplanted into $\alpha 1,3 \mathrm{GT}$-knockout mice failed to induce a substantial immune response relative to mice treated with wild-type cells.
These piglets have the potential to make an important contribution to the field of xenotransplantation, not only by eliminating a major roadblock to successful introduction of foreign tissue, but also, as co-author Yifan Dai points out, by aiding in the identification of other components involved in the triggering of HAR. With this transgenic line established, longterm experiments can begin to assess the potential for safe, rejection-free xenotransplantation, a source of considerable hope for would-be recipients of tissues with limited human donor pools.

\section{-Michael Eisenstein}

\section{'The Pill' for Male Mice}

A recent study found that treating male mice with a sugarlike compound makes them temporarily infertile without affecting hormone levels or causing any other serious side effects. This discovery may move science one step closer to the

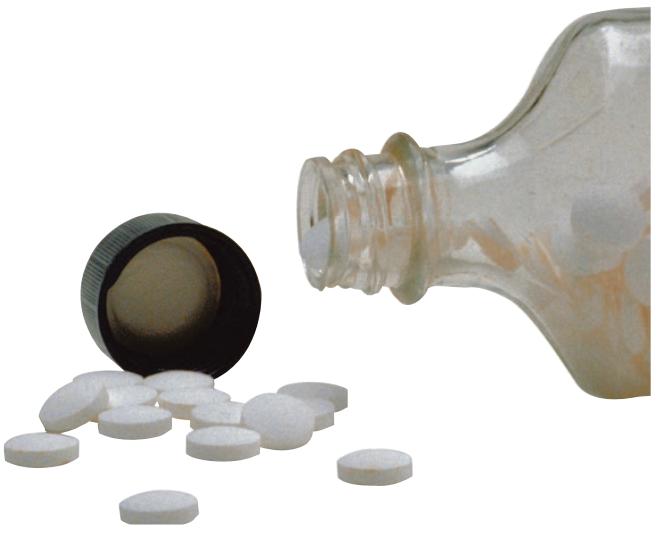

The mice treated with NB-DNJ produced normal levels of sperm, but these were defective and nonfunctional. Most were unable to swim properly because of problems with tail formation. Those sperm that were able to swim were incapable of fertilization due to misshapen or missing acrosomes, the part of the sperm that facilitates penetration of the oocyte.

NB-DNJ has already been through human clinical trials and is a currently approved treatment of the metabolic disorder Gaucher's disease; thus, it may merit quick approval as a male contraceptive. Indeed, Platt's group found that the doses of NB-DNJ necessary to produce infertility were at least ten times less than those used to control Gaucher's disease.

How e ver, much work is still necessary before pharmacists start dispensing NB-DNJ for birth control. According to Aarnoud van der Spoel, the first author on this study, "We are development of a male oral contraceptive.

Most attempts at the development of oral contraceptives for men have focused on the suppression of testosterone production to stymie sperm production. But this approach is complicated and requires androgen replacement because of diminishing endogenous testosterone concentrations.

Now, a group led by Frances M. Platt at the University of Oxford (Oxford, UK) reports that $\mathrm{C} 57 \mathrm{BL} / 6$ males fed small amounts of the alkylated imino sugar $N$ butyldeoxynojirimycin (NB-DNJ) for three weeks were unable to impregnate their female cagemates. Upon discontinuation of NB-DNJ treatment, the male mice regained fertility within four weeks (Proc. Natl. Acad. Sci. USA, 24 December 2002). currently investigating the effects of NBDNJ on the lipid biochemistry and cell biology of the testis."

-Tanja Schub

\section{Silkworms Working for Science? Here's the Skinny}

Japanese researchers have generated transgenic silkworms that produce cocoons containing a protein component of human skin, demonstrating the possibility of using these insects for the mass production of therapeutic human proteins.

The silkworm Bombyx mori has two glands that produce liquid silk; when this liquid comes into contact with the air, it hardens and the silkworm spins it into a cocoon. The cells that compose the silk glands are prolific producers, able to synthesize protein more than a million times 